\title{
ON POSITIVE SOLUTION FOR A CLASS OF QUASILINEAR ELLIPTIC SYSTEMS WITH SIGN-CHANGING WEIGHTS
}

\section{DiAnPENG HE AND ZuOdONG YANG}

Abstract. In this paper, we consider the problem for the existence of positive solutions of quasilinear elliptic system

$$
\left\{\begin{array}{lrl}
-\Delta_{p} u=\lambda a(x) u^{\alpha} v^{\gamma}, & x \in \Omega, \\
-\Delta_{q} v=\lambda b(x) u^{\eta} v^{\beta}, & x \in \Omega, \\
u=v=0, & x \in \partial \Omega,
\end{array}\right.
$$

where the $\lambda>0$ is a parameter, $\Omega$ is a bounded domain in $\mathbb{R}^{N}(N>1)$ with smooth boundary $\partial \Omega$, and the $\Delta_{p} z=\operatorname{div}\left(|\nabla z|^{p-2} \nabla z\right)$ is the $p$-Laplacian operator. Here $a(x)$ and $b(x)$ are $C^{1}$ sign-changing functions that maybe are negative near the boundary. Using the method of sub-super solutions and comparison principle, which studied the existence of positive solutions for quasilinear elliptic system. The main results of the present paper are new and extend the previously known results.

Mathematics subject classification (2010): 35J65, 35J50.

Keywords and phrases: quasilinear elliptic systems, sign-changing weight, positive weak solution.

\section{REFERENCES}

[1] J. Ali, R. Shivaji, Positive solutions for a class of p-Laplacian systems with multiple parameters, J. Math. Anal. Appl., 335 (2007), 1013-1019.

[2] A. CANADA, P. DRABEK, J.L. GAMES, Existence of positive solutions for some problems with nonlinear diffusion, Tras. Amer. Math. Soc., 349 (1997), 4231-4249.

[3] C. CHEN, On positive weak solutions for a class of quasilinear elliptic systems, Nonlinear Anal., 62 (2005), 751-756.

[4] M. ChHetRi, D.D. HAI, R. Shivaji, On positive solutions for classes of p-Laplacian semipositone systems, Discrete Continuous Dyn. Syst., 9, 4 (2003), 1063-1071.

[5] F. CìrsteA, V. RǍdUlESCU, Entire solutions blowing up at infinity for semilinear elliptic systems, J. Math. Pures Appl., 81 (2002), 827-846.

[6] E. Escobedo, M.A. HerRero, Boundedness and blow-up for a semilinear reaction-diffusion system, J. Diff. Eqns., 89 (1991), 176-202.

[7] P. Glansdroff, I. Prigogine, Thermodynamic Theory of Structure, Stability and Fluctuation, Wiley Interscience, London, 1971.

[8] Z. GUO, Some existence and multiplicity results for a class of quasilinear elliptic eigenvalue problems, Nonlinear Anal., 18 (1992), 957-971.

[9] Z. GuO, On the number of positive solutions for quasilinear elliptic eigenvalue problems, Nonlinear Anal., 27, (2)(1996), 229-247.

[10] Z. GUO, Existence and uniqueness of positive radial solutions for a class of quasilinear elliptic equations, Applicable Anal., 47 (1992), 173-190.

[11] D.D. HAI, On a class of quasilinear systems with sign-changing nonlinearities, J. Math. Anal. Appl., 334 (2007), 965-976.

[12] A.V. LAIR, A.W. WOOD, Existence of entire large positive solutions of semilinear elliptic systems, J. Diff. Eqns., 164, (2) (2000), 380-394.

[13] H. Meinhard, Models of Biological Pattern Formation, Academic Press, London, 1982. 
[14] S.H. Rasouli, Z. Halimi, Z. MashHadban, A remark on the existence of positive weak solution for a class of $(p, q)$-Laplacian nonlinear system with sign-changing weight, Nonliear Anal., 73 (2010), 385-389.

[15] Z.D. YANG, Existence of entire explosive positive radial solutions for a class of quasilinear elliptic systems, J. Math. Anal. Appl., 288 (2003), 768-783.

[16] Z. YANG, Q. LU, Nonexistence of positive solutions to a quasilinear elliptic system and blow-up estimates for a quasilinear reaction-diffusion system, J. Compu. Appl. Math., 150 (2003), 37-56.

[17] H. YIN, Z. YANG, New result on the existence of bounded positive entire solutions for quasilinear elliptic systems, Appl. Math. Comput., 190 (2007), 441-448.

[18] H. YIN, Existence result for classes of quasilinear elliptic systems with sign-changing weight, International J. of Non. Science., 10 (2010), 53-60. 